\title{
A 212 SENSITIVITY OF PIV/INTERFACE GRADIOMETRY TO ESTIMATED WALL POSITION
}

\author{
Chuong V. NGUYEN*, Thien D. NGUYEN, John C. WELLS \\ Dept. of Civil \& Environmental Engineering, Ritsumeikan University \\ Noji Higashi 1-1-1, Kusatsu 525-8577 Japan \\ tel(81)77-561-2733, fax(81)77-561-2667 jwells a se.ritsumei.ac.jp \\ * currently, Department of Mechanical Engineering, Monash University, Victoria 3800, Australia \\ Chuong. Nguyen'a,eng.monash.edu.itu
}

ABSTRACT

In recent works, the authors have reported an extension of PIV, called "Interface Gradiometry" (PIV/IG) that, by shearing templates to various degrees, directly measures the instantaneous velocity gradient at a fixed wall. This approach works well when the height of templates is carefully chosen. Our recent generalization of the original PIV/IG (denoted PIV/IG+; Nguyen \& Wells 2006), does not require a template height to be specified a priori for pattern matching, and is thus more widely applicable. It also introduces an image transformation to handle curved boundaries. The present communication considers the sensitivity of PIV/IG+ results to errors in estimated wall position.

Keywords: Particle Image Velocimetry, PIV/IG, velocity profile, velocity gradient, wall shear, curved wall.

\section{INTRODUCTION}

Due to the low level of the velocity signal, and to the rapid deformation of tracer patterns, standard PIV is ill-suited to measurements adjacent to a wall. But nearwall flow is often of interest, and it is worthwhile to tailor image processing to this region. In "Interface Gradiometry" ("PIV/IG"; Nguyen \& Wells 2006a), we sheared image templates, rather than displace them, to directly measure the velocity gradient at a fixed wall, thus mitigating some difficulties with PIV near a wall.

Following Nguyen \& Wells (2006b), the present report treats experimental images, like those in Figure 1 , from a turbulent, locally recirculating flow over a sinusoidal wall in an open channel. We handle the near-wall region as follows. First, we transform image segments to straighten out the boundary; transformed templates are then rectangular, and their lower boundaries correspond to the wall.

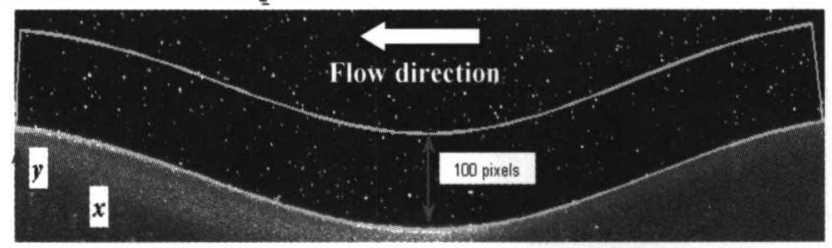

(a) Ø Orthogonal grid generation

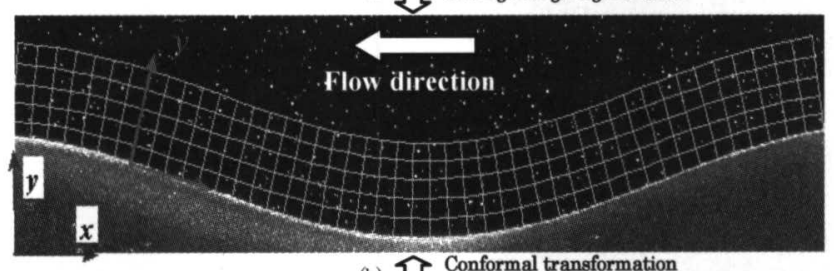

(b) 似

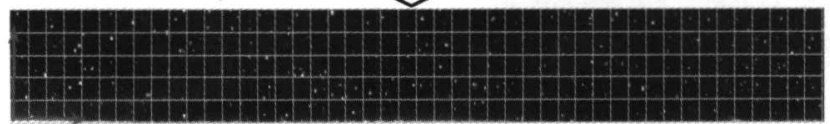

Fig. 1 Orthogonal grid generated by conformal transformation. Given curved boundary (a) of near-wall region in $(\mathrm{x}, \mathrm{y})$ space, orthogonal grids $(\mathrm{b})$ are generated, transformed near-wall image (c) is resampled by $2 \mathrm{D}$ interpolation.
Next, we apply 1D PIV to each horizontal line of pixels within the transformed template, generating a "covariance stack" to be detailed presently. After interpolation and reverse transformation, one obtains, at each point on the boundary, a profile of wall-parallel velocity. To estimate the shear velocity gradient at the wall, which we refer to as "wall shear" or "WS", we fit a line to the covariance stack over a certain "fitting height" adjacent to the wall. We refer to this technique as "PIV/IG+"; an advantage over PIV/IG is that various values of fitting height can be tried during postprocessing, perhaps as part of some local adaptation, without the expensive recomputation of correlation tables. Various other types of post-processing of covariance stacks should also be useful.

\section{CURVED WALL BOUNDARY EXPERIMENT}

The experiment used to test the PIV/IG+ technique has been equipped with double Nd:YAG laser running at 15 $\mathrm{Hz}$ with a $4 \mathrm{~ms}$ interpulse delay. A Kodak ES1.0 (8 bit, $1 \mathrm{~K}^{2}$ interline transfer), synchronized with PIV driver, is operated at $30 \mathrm{fps}$ to capture images at the flow region illuminated by $2-\mathrm{mm}$ thick laser sheet. A $20 \mathrm{~cm}$ x $20 \mathrm{~cm}$ transparent PVC plastic block having a sinusoidal upper surface made by CNC machine was placed on the bottom of a turbulent open-channel flow (Fig. 1). Its wavelength and amplitude were $50 \mathrm{~mm}$ and $2.5 \mathrm{~mm}$, respectively.

\section{OVERVIEW OF PIV/IG+ METHOD}

The PIV/IG+ method consists of 6 steps

1. Identifying the curved wall boundary.

2. Transforming the region of image adjacent to the curved wall into a rectangular region.

3. Calculating the cross correlation between the first and second images to get the covariance profiles ("covariance stacks").

4. Identifying strong peaks in the covariance stacks.

5. Fitting a curve to identified peaks to obtain velocity profile; fitting a straight line to the covariance profiles to obtain wall shear gradient. 
6. Reverse-transforming the velocity profile and velocity gradient to obtain physical values.

Details of each step follow (Nguyen \& Wells, 2006b):

1. Identifying the curved wall boundary

The upper surface of the wavy bed was machined to a specified sinusoid which, scaled appropriately, was overplotted on the raw image and aligned by eye with the boundary. In this paper, we investigate the errors in WS that result from moving this curved wall up and down within a 2 pixels range around the assumed precise boundary (cf. Fig. 2).

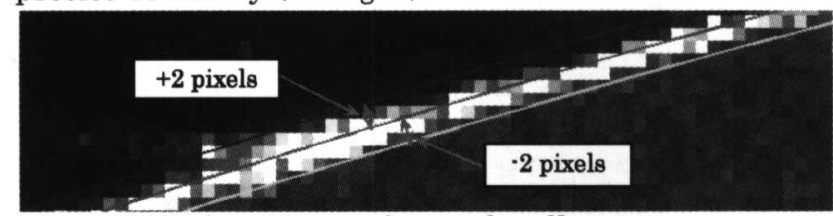

Fig. 2 Shifted positions of curved wall

2. Transforming the region of image adjacent to the curved wall into a rectangular region.

After identifying the wall boundary, an orthogonal grid is generated in a specified strip of the flow region near the wall boundary by applying the free Matlab toolbox "SeaGrid" (Denham, 2000).

3. Cross correlating between the first and second images to yield the "covariance stack".

Like PIV/IG, the PIV/IG+ algorithm requires that wallnormal displacement of tracer particles be less than the particle diameter in the image template. The intensity distribution $I(m)$ along each line $n$ on the template is translated by varying amounts $U$ and "cross"correlated" with the same line in the second image, yielding the covariance $C_{U . n}$ calculated by: $C_{U, n}=\frac{1}{M} \sum_{m=1}^{M}\left[\left(I_{m+U, n}-\bar{I}_{u, n}\right)\left(I_{m, n}^{\prime}-\bar{I}_{n}^{\prime}\right)\right]$

where $\bar{I}_{u, n}$ and $\bar{I}_{n}^{\prime}$ are the mean intensities:

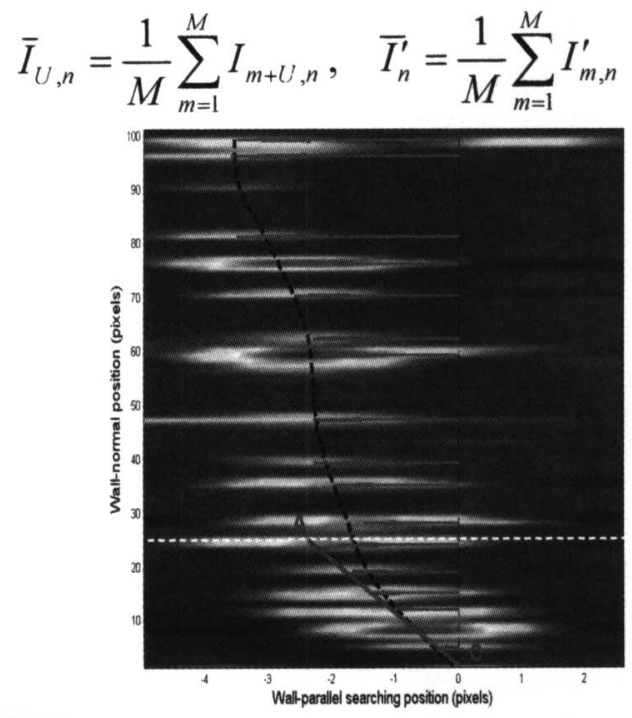

Fig. 3. An instantaneous covariance stack at station 2, near the red axes in Fig. 1. Arrows are identified peaks and dashed curve is interpolated by equation (3). Straight line OA maximizes the sum of covariance over fitting height, taken here to be 25 pixels.
The contour map of $C_{U . n}$ as a function of $U$ (displacement in horizontal axis) and wall-normal position $n$ (vertical position) forms the "covariance stack"; Fig. 3 gives an example.

\section{Identifying strong peaks in covariance stack.}

Strong peaks in the covariance stack are identified as local peaks (in $U$ and $n$ ) within a thresholded region whose threshold value is the mean value of the r.m.s profile of $C U, n$ (cf. Fig. 3)

5. Fitting a curve to identified peaks to obtain velocity profile; fitting a straight line to the covariance stacks to obtain wall shear gradient.

We interpolate a velocity profile $\mathrm{u}(\mathrm{y})$ by summing $\mathrm{u}\left(\mathrm{y}_{\mathrm{n}}\right)$ as weighted by "influence functions" $\Omega$ :

$$
u(y)=\frac{\sum_{n=1}^{N} u\left(y_{n}\right) \Omega\left(y, y_{n}\right)}{\sum_{n=1}^{N} \Omega\left(y, y_{n}\right)}
$$

in which $\Omega$ is taken here to be a Gaussian: $\Omega\left(y, y_{n}\right)=\exp \left[-\frac{\left(y-y_{n}\right)^{2}}{\sigma^{2}}\right]$. The no-slip condition is satisfied by adding a reflection of velocity profile as $u(-y)=-u(y)$.

Only the near-wall part of the covariance stack is used for estimating wall shear gradient; we refer to this region as the "fitting height", which will take values of 15,25 , and 35 pixels herein, gradient. The line of best fit OA, exemplified in Fig. 3. and Fig. 4, is that which maximizes the sum of the interpolations of covariance values along the line.

6. Reverse-transforming the velocity profile and velocity gradient to obtain physical values.
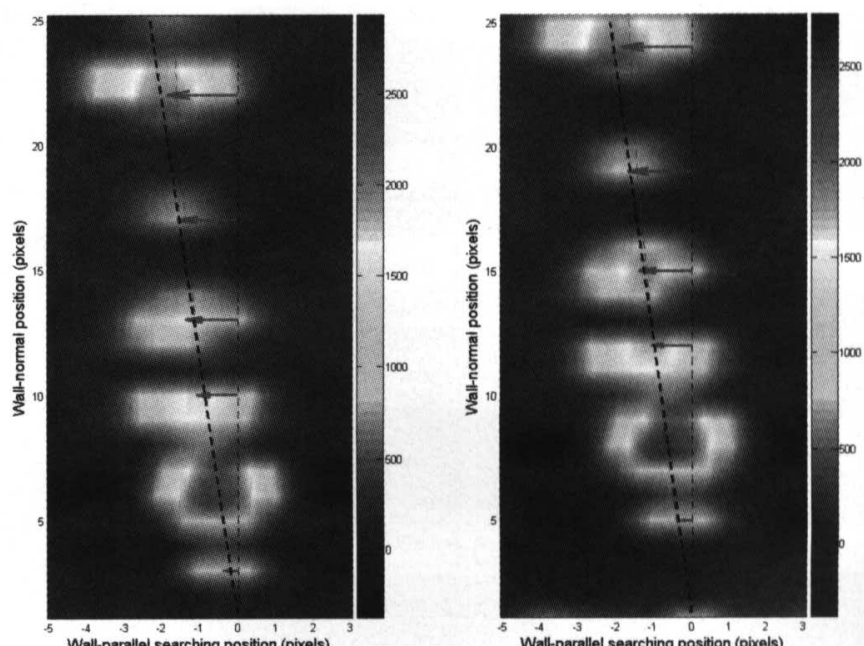

Fig. 4. Close-up of covariance stack of Fig. 3. Red curve is interpolated velocity profile with $\sigma=10$, while dashed black line corresponds to $\mathrm{OA}$ in Fig. 3 (a) true wall position (b) wall shifted up by 2 pixels. 


\section{SENSITIVITY OF RESULTS TO WALL POSITION}

In the present communication, we examine the sensitivity of wall shear, as measured by PIV/IG+, to shifts in assumed wall position with respect to the "true" boundary position determined in step 1 and indicated in Figure 2. Fig. 4(a) is a closeup of the region in Fig. 3 below 25 pixels from the wall. In (b), the assumed wall position is shifted up 2 pixels. The dashed lines maximize the sum of covariance in the two cases. By chance, there is a tracer particle near the top of the template, and the lines of best fit appear to be "locked in" by the corresponding covariance peaks. This is analogous with the original PIV/IG, where correlation peaks near the top of the template were found (Nguyen \& Wells, 2005) to dominate estimates of WS. At their lower ends, the lines are constrained to pass through the origin at the wall. Roughly speaking then, one expects the slope of the line of best fit to vary inversely with distance from the assumed wall position to the dominant correlation peak near the top of the fitting region.

In Nguyen \& Wells (2006b), we suggested that the covariance stacks compress nearly all information available on inter-image tangential tracer displacement near the boundary, and that in a PIV run it will be feasible and justified to save all instantaneous stacks at each boundary point for post-processing. The most basic of such post-processing is, following Meinhart et al. (2000), to compile the time-averaged covariance stack (Fig. 5), which may then be fitted with lines near the wall to estimate time-averaged WS. Figure 6 compares the time-average wall shear measured in this way for fitting heights of 15,25 , and 35 pixels, and for shifts in wall position up to 2 pixels. At this boundary location, shortly following reattachment, the mean velocity profile is strongly convex near the wall. As a result, the data depend strongly on the fitting height; in particular for $y_{w}=0$, i.e. for the true wall position, the measured value of average $\partial \mathrm{U} / \partial \mathrm{Y}$ increases some $50 \%$ as fitting height is varied from 35 to 15 pixels.


Wall-parallel searching position (pixels) Wall-parallel searching position (pixels)

Fig. 5 Time-averaged covariance stack at station 2 , with interpolated velocity profile obtained by Gaussian $(\sigma=$ 10) (a) precise wall position (b) 2 pixels higher than precise wall position.
Fig. 5(a) suggests that to resolve the true value of WS, one should take the fitting height as low as 4 pixels, which is feasible for the time-averaged covariance stack though not for instantaneous stacks). Consider next the effect of shifts in assumed wall position. The overall slopes of the curves become steeper, i.e. the measured value of $\partial \mathrm{U} / \partial \mathrm{Y}$ is more sensitive to errors in wall position, as fitting height is decreased.

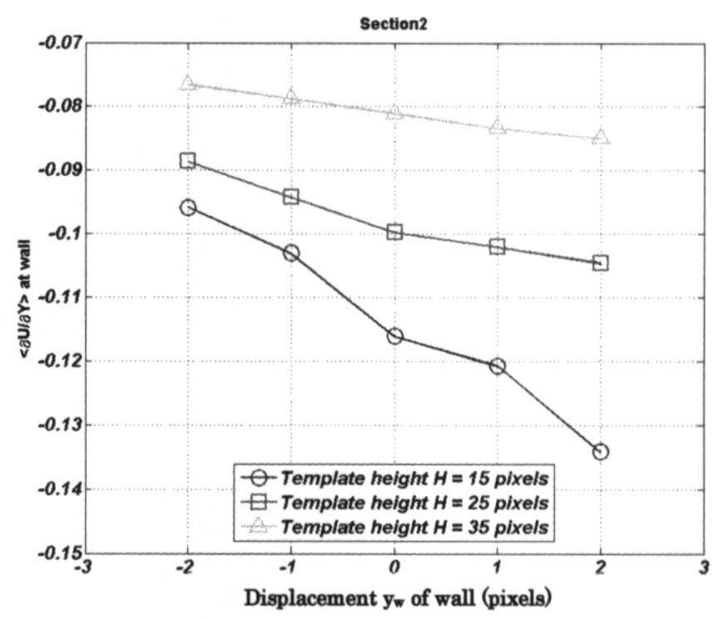

Fig. 6. $\partial \mathrm{U} / \partial \mathrm{Y}$ at wall by fitting line to time averaged covariance stack, versus shift in wall position.

This trend is also observed in Fig. 7, which is an analogous plot of the average and r.m.s. fluctuation of $\partial \mathrm{U} / \partial \mathrm{Y}$ determined from instantaneous covariance stacks.

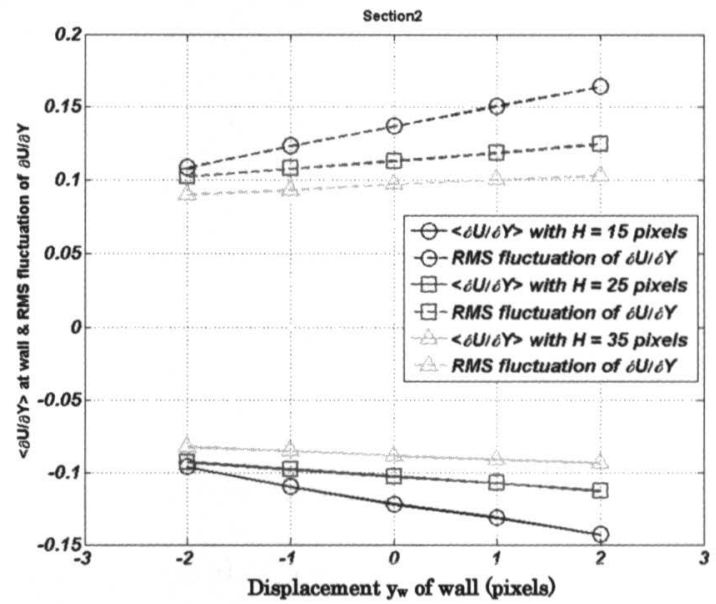

Fig. 7. Time averaged $\partial \mathrm{U} / \partial \mathrm{Y}$ and r.m.s fluctuation of $\partial \mathrm{U} / \partial \mathrm{Y}$ at wall by fitting lines to instantaneous covariance stacks.

The differences $\Delta$ in data between $y_{w}=+1$ and -1 pixels in the latter figure have been used to evaluate the sensitivity of $\partial \mathrm{U} / \partial \mathrm{Y}$ to $y_{w}$, denoted $s$, and a nondimensional form $\widetilde{S}$, as follows

$$
s=\frac{\Delta\left(\frac{\partial U}{\partial Y}\right)}{\Delta y_{w}} \quad \tilde{s}=\frac{\Delta\left(\frac{\partial U}{\partial Y}\right) H}{\left.\frac{\partial U}{\partial Y}\right|_{y_{w}=0} \Delta y_{w}}
$$

where $H$ is the fitting height, and where $\partial \mathrm{U} / \partial \mathrm{Y}$ stands here for both the time-average $\langle\partial \mathrm{U} / \partial \mathrm{Y}\rangle$ and for the 
r.m.s. fluctuation of $\partial \mathrm{U} / \partial \mathrm{Y}$. The values of nondimensional sensitivity $\tilde{s}$ are plotted in Fig. 8 versus template height. There is some variation, notably for a fitting height of 15 pixels, which suggests that length scales other than $H$ also affect $s$; perhaps the higher density of tracer particles inferred from Fig. 5 to occur at $Y=10$ pixels, and/or the viscous length scale $u_{t} / v$, are relevant.

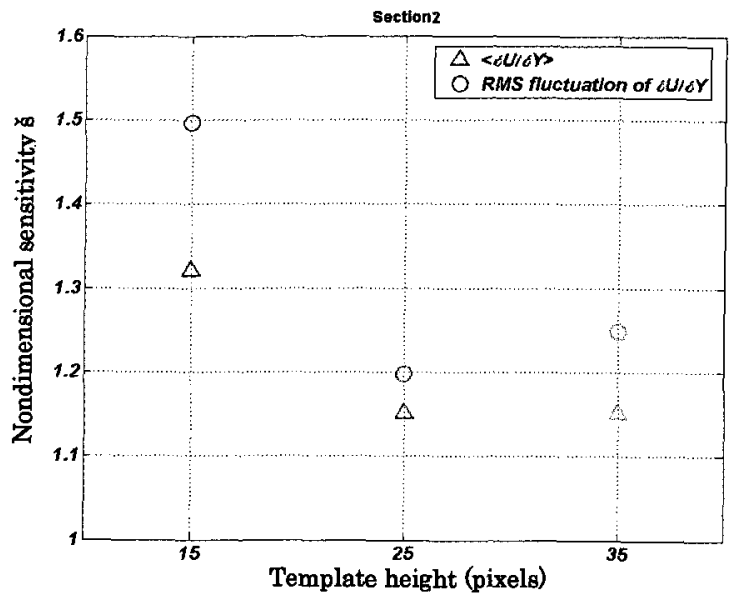

Fig. 8. Normalized sensitivity $\widetilde{\boldsymbol{S}}$ of time-averaged $\partial \mathrm{U} / \partial \mathrm{Y}$ and r.m.s fluctuation $\partial \mathrm{U} / \partial \mathrm{Y}$ at wall to the shift in wall position.

\section{CONCLUSION:}

Despite the variation just mentioned, the values of $\widetilde{s}$ lie in the fairly narrow range $1.25+/-0.10$ for $\langle\partial \mathrm{U} / \partial \mathrm{Y}\rangle$, and $1.35+/-0.15$ for $(\partial U / \partial Y)_{\text {rms. Given some uncertainty }}$ $\Delta y_{w}$ in wall position, perhaps based on inspection of the images, one might estimate the relative error in $\partial \mathrm{U} / \partial \mathrm{Y}$ according to

$$
\frac{\Delta\left(\frac{\partial U}{\partial Y}\right)}{\left.\frac{\partial U}{\partial Y}\right|_{y_{w}=0}}=\tilde{s} \frac{\Delta y_{w}}{H}
$$

in which $\widetilde{S}$ could be assigned a value from the upper end of the ranges quoted above; continued investigation is desirable to confirm that these values are valid for other experimental conditions. But since $\widetilde{s}$ is unlikely to ever be less than 1.0 , the principal conclusion of this work is that when imposing the no-slip condition to help infer WS, results are rather sensitive to the uncertainty $\Delta y_{w}$ in wall position. Thus great care should be taken to locate the wall as precisely as possible, to subpixel accuracy if possible.

We have also mentioned the importance of the fitting height $H$, which roughly corresponds to an averaging height of the near-wall velocity profiles. In principle, one would like to specify the minimal value of $H$ compatible with the experimental tracer density, but the estimated error due to wall position, perhaps estimated as in the preceding paragraph, may also turn out to be a limiting factor.

\section{REFERENCES:}

Denham, Charles R. (2000), SeaGrid Orthogonal Grid Maker for Matlab, http://woodshole.er.usgs.gov/staffuages/cdenham/public html/seagrid/seagrid html

Meinhart, C. D., Wereley S. T. and Santiago J. G. (2000), A PIV Algorithm for Estimating Time-Averaged Velocity Fields, Journal of Fluids Engineering 122: 285. 289

Nguyen, C. V. and Wells, J.C., (2005) Know thy validator: What is the transfer function for PIV/interface gradiometry?, EUROMECH 469, (Dresden Germany, Oct. 2005).

Nguyen, C. V. and Wells, J.C. (2006a), Direct measurement of fluid velocity gradients at a wall by PIV image processing with stereo reconstruction, Journal of Visualization, Paper Number: 05-027; adapted from; Nguyen et al., Proc. Int. Conf. on Advanced Optical Diagnostics in Fluids, Solids and Combustion (Tokyo, Japan, Dec. 2004), V0045

Nguyen, C. V. and Wells, J.C. (2006b), Development of PIV/Interface gradiometry to handle low tracer density and curved walls, FEDSM2006-98568 Proceedings of FEDSM2006 European Fluids Engineering Summer Meeting (Miami, USA, June 2006) 